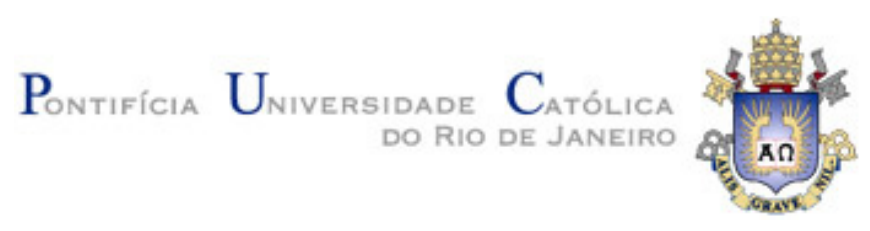

Felipe Albuquerque Portella

Um serviço de captura e acesso para espaços ativos

Dissertação apresentada como requisito parcial para obtenção do título de Mestre pelo Programa de PósGraduação em Informática da PUC-Rio.

Orientador: Renato Cerqueira

Rio de Janeiro, abril de 2008 


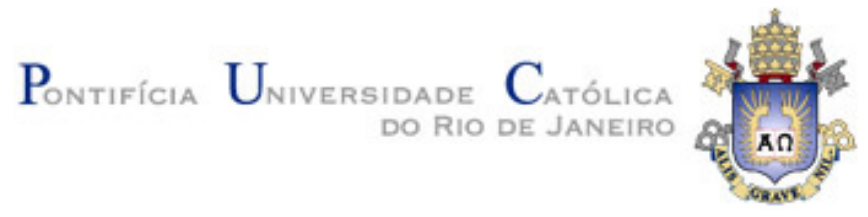

Felipe Albuquerque Portella

\section{Um serviço de captura e acesso para espaços ativos}

Dissertação apresentada como requisito parcial para obtenção do título de Mestre pelo Programa de PósGraduação em Informática da PUC-Rio. Aprovada pela Comissão Examinadora abaixo assinada.

Renato Cerqueira Orientador Departamento de Informática - PUC-Rio

Prof. Luiz Fernando Gomes Soares Departamento de Informática - PUC-Rio

Prof. Markus Endler Departamento de Informática - PUC-Rio

Prof. José Eugenio Leal Coordenador Setorial do Centro Técnico Científico - PUC-Rio 
Todos os direitos reservados. É proibida a reprodução total ou parcial do trabalho sem autorização da universidade, do autor e do orientador.

\section{Felipe Albuquerque Portella}

Graduou-se como Bacharel em Informática na Pontifícia Universidade Católica do Rio de Janeiro em 2003. Atuou como analista de sistemas no TecGraf, laboratório de computação gráfica da PUC-Rio, onde desenvolveu diversos projetos para o CENPES, Centro de Pesquisas da Petrobras. Atualmente pesquisa e desenvolve soluções para a área Exploração e Produção (E\&P) da Petrobras, da qual é funcionário. Possui interesse nas áreas de Engenharia de Software, Sistemas Distribuídos e Computação Ubíqua.

Ficha Catalográfica

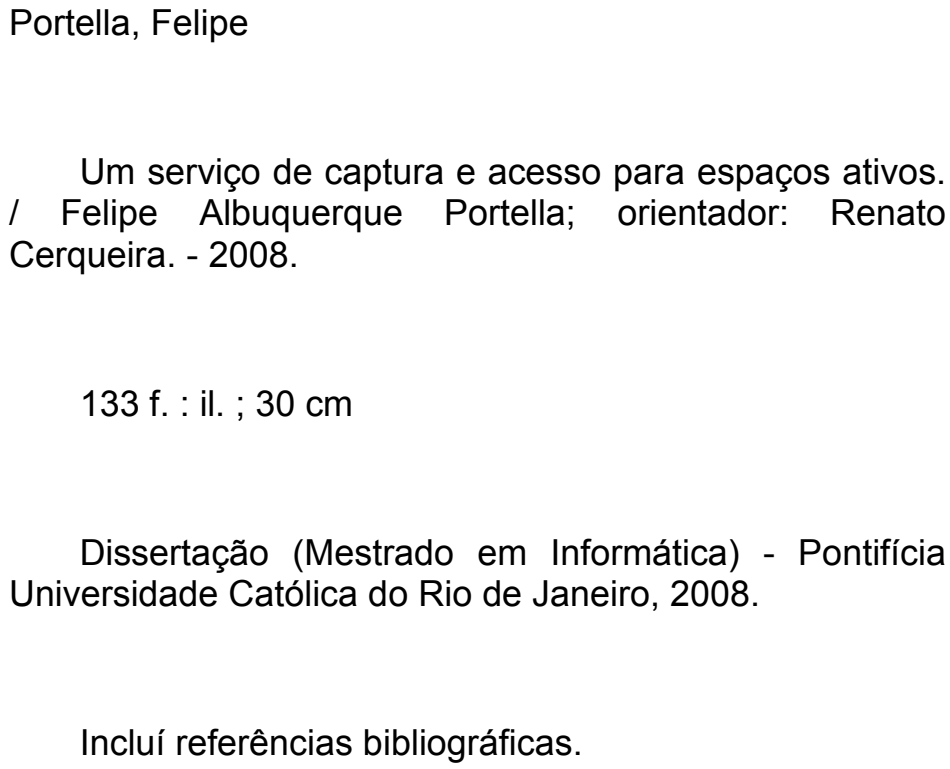

Um serviço de captura e acesso para espaços ativos. I Felipe Albuquerque Portella; orientador: Renato Cerqueira. - 2008.

133 f. : il. ; $30 \mathrm{~cm}$

Dissertação (Mestrado em Informática) - Pontifícia Universidade Católica do Rio de Janeiro, 2008.

Incluí referências bibliográficas.

1. Informática - Dissertações. 2. Sistemas Distribuídos. 3. Computação Ubíqua. 4. Captura \& Acesso. I. Cerqueira, Renato. II. Pontifícia Universidade Católica do Rio de Janeiro. Departamento de Informática. III. Título. 


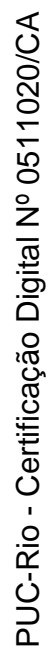

Aos meus pais Ledir e Francisco 


\section{Agradecimentos}

Aos meus pais pelo constante amor, carinho e apoio em todos os momentos importantes da minha vida.

Ao meu orientador Renato Cerqueira pela sua confiança, orientação e amizade, ajudando a tornar este trabalho interessante e divertido.

Aos professores Luiz Fernando Gomes Soares e Markus Endler por participarem da Banca Examinadora.

À minha namorada Rapha por todo seu amor, incentivo, compreensão e paciência, além das inúmeras revisões de textos.

Por fim, gostaria de agradecer à Microsoft Research pelos recursos para o desenvolvimento deste trabalho. À PUC-Rio e ao TecGraf pelo apoio financeiro ao longo de todo o mestrado. Bem como à Petrobras por seu investimento de tempo para a minha formação. Auxílios importantes, sem os quais este trabalho não poderia ter sido realizado. 


\section{Resumo}

Portella, Felipe. Um serviço de captura e acesso para espaços ativos. Rio de Janeiro, 2008. 133p. Dissertação de Mestrado - Departamento de Informática, Pontifícia Universidade Católica do Rio de Janeiro.

Uma das áreas de grande destaque dentro da Computação Ubíqua é a de aplicações multimídia para Captura \& Acesso (C\&A). Essas aplicações permitem a captura de uma experiência ao vivo, normalmente em ambientes instrumentados, para seu acesso no futuro. Dessa forma transfere-se para os computadores a responsabilidade de gravar o evento, permitindo que as pessoas tenham seu foco de atenção na compreensão e interpretação da experiência em si, sem se preocupar com a tarefa de registrar a informação. A literatura apresenta muitas ferramentas que permitem a geração automática de documentos multimídia como resultado da captura de um evento, e esses mesmos documentos são usados como base para navegação e busca sobre o conteúdo armazenado. Tipicamente, essas ferramentas de C\&A geram documentos que oferecem uma navegação com base apenas na linha de tempo (timeline) do evento registrado. Esta dissertação propõe uma infraestrutura genérica de C\&A, baseada em serviços reutilizáveis e intercambiáveis, que explora os recursos oferecidos pela linguagem NCL para investigar novos paradigmas na engenharia de documentos produzidos por aplicações de C\&A, através da estruturação dos documentos em modelos conceitual, navegacional e de apresentação. Utilizamos a linguagem NCL tanto para registrar o sincronismo entre as diferentes mídias gravadas, quanto para gerar diferentes formas de navegação e apresentação do conteúdo gravado. Os modelos de navegação e apresentação são gerados com base em metadados fornecidos pelo usuário ou extraídos automaticamente do conteúdo gravado.

\section{Palavras-chave}

Aplicações de Captura e Acesso; Documentos Multimídia; Computação Ubíqua; Espaços Ativos; Aplicações Multimídia; NCL. 


\section{Abstract}

Portella, Felipe. A capture and access service for active spaces. Rio de Janeiro, 2008. 133p. Master Thesis - Department of Informatics, Pontifical Catholic University of Rio de Janeiro.

One of the areas of most evidence in Ubiquitous Computing is multimedia applications of Capture \& Access (C\&A). This kind of application allows the capture of a live experience, usually in smart rooms, for future access. In this way, the responsibility for recording the event is transferred to the computing infrastructure, allowing users to focus their attention in the comprehension and interpretation of the experience itself, without worrying about registering the information. The literature presents many software systems allowing the automatic generation of hypermedia documents as the result of an event capture, using the same documents as the basis for navigation and search of the archived content. Typically these C\&A applications generate documents that offer only a timeline navigation of the captured event. This dissertation proposes a general C\&A infrastructure, based on reusable and interchangeable services, which explores the features offered by the NCL language (standard language of the Brazilian Digital TV) to investigate new paradigms in C\&A documents engineering. This is accomplished by structuring the generated documents in conceptual, navigation and presentation models. The NCL language is used to represent the synchronism between the different recorded media as well as to generate different ways to navigate and present the recorded content. These models of navigation and presentations are based on metadata provided by the user or automatically extracted from the recorded content.

\section{Keywords}

Capture \& Access Applications; Hypermedia Documents; Ubiquitous Computing; Active Spaces; Multimedia Applications; NCL. 


\section{Sumário}

1 Introdução 14

1.1. Posicionamento e Motivação 15

1.2. Objetivos e Contribuições 16

$\begin{array}{ll}\text { 1.3. Estrutura da Dissertação } & 17\end{array}$

2 Computação Ubíqua $\quad 19$

2.1. Interfaces Naturais 21

2.2. Computação Ciente de Contexto 22

2.3. Captura \& Acesso 24

2.3.1. Espaço de Projeto 25

2.3.2. Fases de Captura e Acesso 26

2.3.3. Desafios em Captura e Acesso 27

2.3.4. Ambientes de Captura \& Acesso 29

3 Trabalhos Relacionados 31

3.1. eClass/Classroom 2000

3.2. InCA-SERVE 32

3.2.1. $x \ln C A$

3.2.2. eMeet 33

3.2.3. iClass $\quad 35$

3.3. ConferenceXP 36

3.3.1. Classroom Presenter 38

3.3.2. ConferenceXP Archive Service 39

3.3.3. ConferenceXP Web Viewer 39

3.3.4. ConferenceXP Archive Transcoder 41

3.3.5. ConferenceXP to Windows Media Gateway 41

3.4. Considerações sobre os Sistemas 42

4 Abordagem Proposta $\quad 45$

$\begin{array}{ll}\text { 4.1. SpeedCar } & 47\end{array}$ 
4.2. CASIn 48

4.2.1. CASWeb 48

4.2.2. SpeedCar Launcher Service $\quad 49$

4.2.3. Post-Production Service 49

4.2.4. Transformadores 53

4.2.4.1. Transformador de Detecção de Contextos Simples54

4.2.4.2. Transformador de Detecção de Contextos Baseado em Padrões 55

4.2.4.3. Transformador de Detecção de Contextos em Slides Não Adjacentes

4.2.4.4. Transformador de Remoção de Transições Curtas 56

4.3. Limitações da Arquitetura Proposta 57

5 Aspectos de Implementação 61

5.1. Modelo de Comunicação 61

5.2. Componentes Desenvolvidos 63

5.2.1. SpeedCar 63

5.2.1.1. SpeedCar Áudio 65

5.2.1.2. SpeedCar D-Link Video 66

5.2.1.3. SpeedCar PowerPoint 66

5.2.2. CASIn 67

5.2.2.1. CASWeb 68

5.2.2.2. SpeedCar Launcher Service 71

5.2.2.3. Post-Production Service 72

5.2.2.4. CAS Filters 75

$\begin{array}{ll}\text { 5.2.3. Acesso } & 77\end{array}$

6 Experimentos $\quad 80$

6.1. Encontro de Python Científico na Petrobras 80

6.1.1. Configurações e Problemas de Infra-Estrutura 81

6.1.2. Primeira Palestra 83

6.1.3. Segunda palestra $\quad 84$

6.1.4. Terceira Palestra 84

6.1.5. Quarta Palestra 84 
6.1.6. Quinta Palestra 84

6.2. Aulas de Seminários de Sistemas Distribuídos 85

6.2.1. Configurações e Problemas de Infra-Estrutura 85

6.2.2. Primeiro Seminário 86

6.2.3. Segundo Seminário 88

6.3. Avaliação Geral dos Experimentos Realizados 90

7 Considerações Finais $\quad 92$

7.1. Trabalhos Futuros 93

7.1.1. Novas Funcionalidades $\quad 93$

7.1.2. Temas de Pesquisa 95

$\begin{array}{ll}\text { Referências Bibliográficas } & 98\end{array}$

$\begin{array}{ll}\text { Apêndice A CAS XML Schema } & 106\end{array}$

Apêndice B Primeiro Evento do Seminário de Sistemas Distribuídos 109

B.1 SpeedCar Audio XML 109

B.2 SpeedCar D-Link XML (Câmera 1) 110

B.3 SpeedCar D-Link XML (Câmera 2) 110

B.4 SpeedCar PPT XML 111

B.5 PresentationInfo XML 113

B.6 NCL Gerada para o Evento 117 


\section{Lista de figuras}

Figura 2.1: Alguns dispositivos ubíquos atuais (HP Tablet PC, Apple iPhone, Logitech IO Pen, Microsoft Surface).

Figura 2.2: Taxonomia dos problemas de pesquisa em Computação Ubíqua [Saty01].

Figura 2.3: Reconhecimento de gestos para a interação em um jogo [Truy05]. 21

Figura 2.4: Modelo de contexto 3D [SATT99]. 23

Figura 3.1: Arquitetura do sistema eMeet/SERVE [Eleu02]. 34

Figura 3.2: Visualização de uma sessão pelo eMeet/SERVER a partir dos dados capturados pelo eMeet/InCA [Eleu02]. 34

Figura 3.3: Apresentação SMIL da sessão capturada com o iClass no Real Player [CARP03]. 35

Figura 3.4: Arquitetura do ConferenceXP [BCHM04]. 37

Figura 3.5: Visão do professor em uma aula com o Classroom Presenter [Clas08].

Figura 3.6: ConferenceXP Web Viewer. 39

Figura 4.1: Visão geral da arquitetura do CAS com seus principais elementos. 46

Figura 4.2: Workflow interno do Post-Production Service com as entradas e saídas $\begin{array}{ll}\text { de cada componente. } & 50\end{array}$

Figura 4.3: Nós e elos num documento hipermídia comum [NSRB07]. 52

Figura 4.4: Nós, elos e nós de composição (contextos) [NSRB07]. 53

Figura 5.1: Estrutura de um serviço WCF [Chap07]. 62

Figura 5.2: Arquitetura detalhada do CASIn. $\quad 68$

Figura 5.3: Página inicial do CASWeb. $\quad 69$

Figura 5.4: Tela de gravação de um evento no CASWeb. $\quad 70$

Figura 5.5: Tela de entrada de contexto manual no CASWeb. 71

Figura 5.6: Menu de seleção para navegação por contexto da terceira palestra do evento de Python (detalhado na subseção 6.1.4) no Ginga NCL Emulator. 78

Figura 5.7: Reprodução da terceira palestra do evento de Python (detalhado na subseção 6.1.4) no Ginga NCL Emulator com três vídeos em sincronia com os slides. 
Figura 6.1: Sumário da apresentação com oito tópicos segundo a concepção do autor. 
"A simplicidade é a derradeira sofisticação." Leonardo da Vinci 